\title{
Characterization of carboxylate
} producing microbial communities

\author{
Nathaniel W. Fortney*a, Kevin A. Walters ${ }^{a, b}$, Matthew J.
} Scarborough $^{c}$, Kevin S. Myers ${ }^{a}$, Abel T. Ingle ${ }^{d}$, Paula R. F. Rosa $^{a, e}$, Timothy J. Donohue ${ }^{a, b}$, Daniel R. Noguera ${ }^{a, d}$

* nfortney@wisc.edu

a Great Lakes Bioenergy Research Center, University of WisconsinMadison, Madison, WI, USA; ${ }^{b}$ Department of Bacteriology, University of Wisconsin-Madison, Madison, WI, USA; ' Department of Civil and Environmental Engineering, University of Vermont, Burlington, VT, USA; ${ }^{d}$ Department of Civil and Environmental Engineering, University of Wisconsin-Madison, Madison, WI, USA; e Department of Chemical Engineering, Federal University of São Carlos, São Carlos, SP, Brazil

\section{HIGHLIGHTS:}

- Enriched for Clostridiales and Lactobacillales putatively involved in production of industrially relevant carboxylates, independent of growth feedstock.

- Alteration of operational conditions of bioreactors, e.g. increased temperature, can select for a different profile of fermentation products.

- Two recently described MCFA-producing strains, Ca. Weimeria bifida, and $\mathrm{Ca}$. Pseudoramibacter fermentans [1], enriched from lignocellulosic residues, have also been identified as prominent community members in the current experiments utilizing different feedstocks.

BACKGROUND: Carboxylic acids, including succinic acid, lactic acid, and medium chain fatty acids (MCFAs), are valuable chemicals that can be produced from a variety of industrial residues by fermentative microbial communities. Residues from lignocellulosic biorefineries (conversion residue; $C R$ ), starch ethanol plants (thin stillage; TS), and the dairy industry (ultra-filtered milk permeate; UFMP) are examples of carbon-rich, low-value co-products that are typically sent to anaerobic digesters for biogas [2] or sold as animal feed [3]. Diverse product formation from primary feedstocks can help offset operating costs, reduce the selling point of the primary products (e.g. biofuel), and ultimately make these industries more economically viable [4].

RESULTS \& DISCUSSION: The CR-fed bioreactor was stable for over 100 $\mathrm{d}$ and produced primarily $\mathrm{C} 4$ and $\mathrm{C} 6$ fatty acids. The UFMP-fed bioreactor initially produced $\mathrm{C} 6$ and $\mathrm{C} 8$ fatty acids, and then, shifted to producing primarily butyrate (C4). TS-fed bioreactors produced a mixture of C5-C8 fatty acids. Decreasing the retention time in the TS-fed bioreactor induced a shift to succinate production. Furthermore, increasing temperature of the 
TS-fed bioreactor to $55^{\circ} \mathrm{C}$ induced shifts to lactic and propionic acid as the primary fermentation products. Identification of microorganisms using $16 \mathrm{~S}$ rRNA gene amplicon sequencing revealed high abundance of Clostridiales and Lactobacillales in communities enriched on CR, TS, and UFMP. The UFMP bioreactor enriched for organisms related to the recently defined Agathobacter genus [5] within the Lachnospiraceae. In the TS reactors, Prevotella (phyl. Bacteroidetes), Lactobacillus-relatives, and the Clostridia Pseudoramibacter were prominent under MCFA-producing conditions and a disappearance of Butyrivibrio occurred when retention time decreased (Figure 1). The Clostridia were absent from the thermophilic TS bioreactor, and Actetobacter were abundant in addition to the Lactobacillus-relatives. Metagenomic analyses of these microbial communities is underway

CONCLUSION: The ability to enrich for carboxylate-producing taxa from the same inoculum source, but fed variety of organic-rich substrates is promising for the future of sustainable production of commodity chemicals, and is not tied to the residues of a single industry. The ability to adjust bioreactor conditions to control for certain microbial communities could help industries adapt to switches on prices or demand for chemicals produced by fermentation.

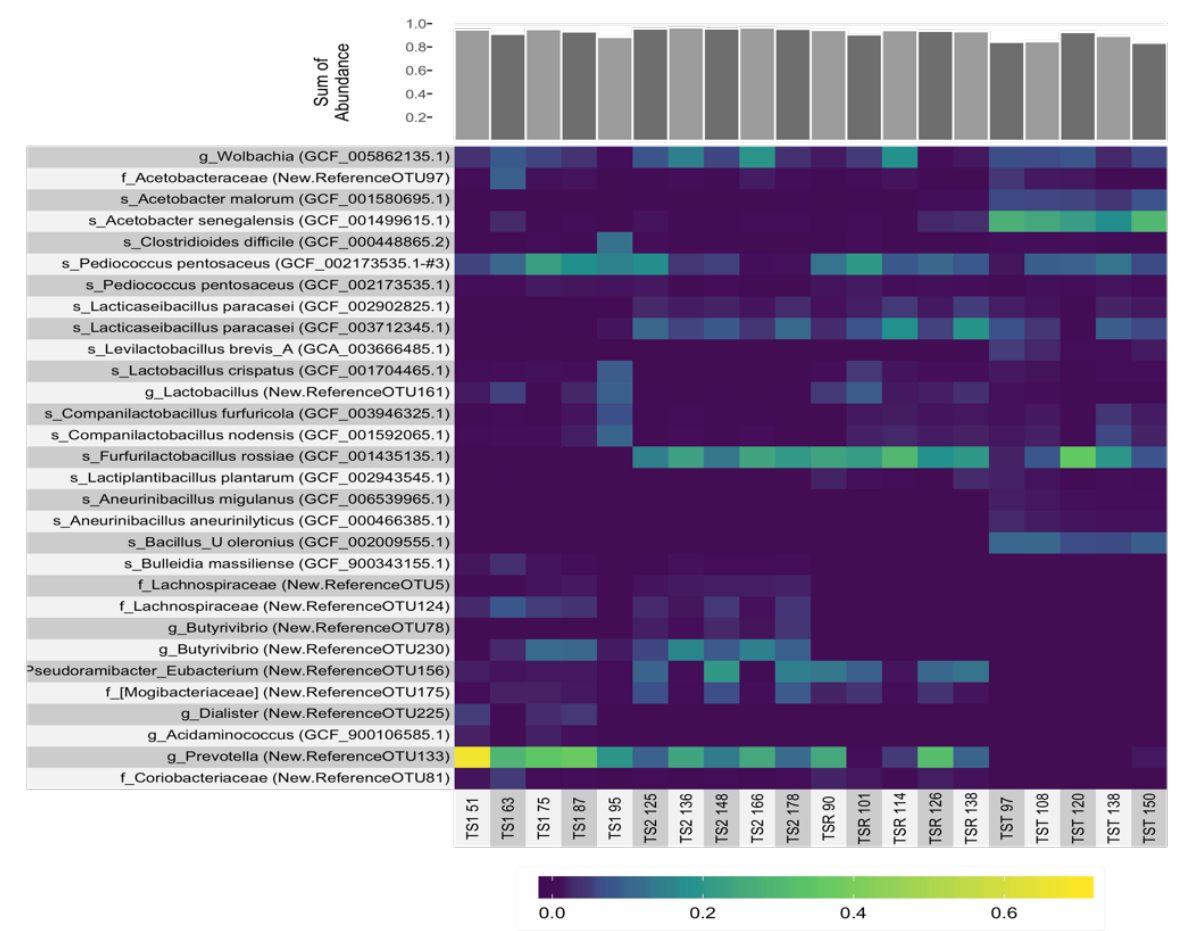

Figure 1. 16S rRNA gene amplicon based operational taxonomic units with greater than $1 \%$ relative abundance. OTUs represent microbial community during periods of stable bioreactor conditions (ca. $50 \mathrm{~d}$ ) for four different bioreactor operational conditions. TS1, unaltered thin stillage, $\mathrm{pH} 5.5,35^{\circ} \mathrm{C}$,

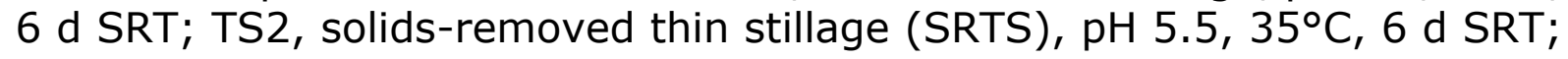
TSR, SRTS, pH 5.5, $35^{\circ} \mathrm{C}, 1 \mathrm{~d} \mathrm{SRT}$; TST, SRTS, pH 4.5-5.0, 55 ${ }^{\circ} \mathrm{C}$, $6 \mathrm{~d}$ SRT. 
This material is based upon work supported by the DOE Great Lakes Bioenergy Research Center, U.S. Department of Energy, Office of Science, Office of Biological and Environmental Research under Award Number DESC0018409, the National Institute of Food and Agriculture (USDA 201767003-25055), and the National Dairy Council (Project \#AA68952).

\section{REFERENCES}

1. Scarborough MJ, Myers KS, Donohue TJ, Noguera DR. 2020. Medium-Chain Fatty Acid Synthesis by "Candidatus Weimeria bifida" gen. nov., sp. nov., and "Candidatus Pseudoramibacter fermentans" sp. nov. Appl Environ Microbiol 86:2796.

2. Gerbrandt $K$, Chu PL, Simmonds A, Mullins KA, MacLean HL, Griffin WM, Saville BA. 2016. Life cycle assessment of lignocellulosic ethanol: a review of key factors and methods affecting calculated GHG emissions and energy use. Curr Opin Biotechnol 38:63-70.

3. Lupitskyy R, Staff C, Satyavolu J. 2015. Towards integrated biorefinery from dried distillers grains: Evaluation of feed application for Co-products. Biomass and Bioenergy 72:251-255.

4. Scarborough MJ, Lynch G, Dickson M, McGee M, Donohue TJ, Noguera DR. 2018. Increasing the economic value of lignocellulosic stillage through medium-chain fatty acid production. Biotechnol Biofuels 11:1-17.

5. Rosero JA, Killer J, Sechovcová H, Mrázek J, Benada O, Fliegerová K, Havlík J, Kopečný J. 2016. Reclassification of Eubacterium rectale (Hauduroy et al. 1937) Prévot 1938 in a new genus Agathobacter gen. nov. as Agathobacter rectalis comb. nov., and description of Agathobacter ruminis sp. nov., isolated from the rumen contents of sheep and cows. Int J Syst Evol Micr 66:768773. 